\title{
Utilisations détournées du protoxyde d'azote
}

\section{Abuse of nitrous oxide}

\section{Claire BOUTRON ${ }^{(1)}$, Monique MATHIEU-NOLF(1), Nicolas PETY(1), Marc DEVEAUX ${ }^{(2)}$}

(1) Centre Antipoison, 5, avenue Oscar Lambret - 59037 LILLE Cedex (2) Institut de Médecine Légale, place Théo Varlet - 59000 LILLE

*Auteur à qui adresser la correspondance : Dr Monique MATHIEU-NOLF, Centre Antipoison, 5, avenue Oscar Lambret - 59800 LILLE - Tél Secrét. : 0320444799 - Fax : 0320445628 e-mail : mmathieu@chru-lille.fr

(Reçu le 19 juillet 2000 ; accepté le 10 août 2000)

\section{RÉSUMÉ}

Le protoxyde d'azote, gaz médical utilisé de longue date en médecine pour ses propriétés anesthésiques et analgésiques, et en industrie alimentaire comme gaz propulseur, fait aujourd'hui l'objet d'une utilisation détournée qui semble se répandre en France, comme c'est déjà le cas depuis plus de vingt en Angleterre ou aux Etats-Unis. Afin de sensibiliser le corps médical, le Centre Antipoison de Lille a réalisé une synthèse sur les données de l'intoxication aiguë et chronique au protoxyde d'azote et sur sa détection et son dosage dans les milieux biologiques.

La synthèse scientifique a été réalisée à partir d'une recherche bibliographique utilisant MedLine. Par ailleurs, des contacts avec une association Lilloise de ravers ont permis d'obtenir des connaissances plus pratiques sur les lieux, quantités et fréquence de cette consommation.

Le protoxyde d'azote est conditionné pur sous forme liquide, ou en mélange équimoléculaire avec l'oxygène (MEOPA) sous forme gazeuse, dans des bouteilles de capacités variables selon le laboratoire. Leur couleur dépend de l'utilisation, médicale ou industrielle, à laquelle le produit est destiné. Il se présente également sous forme de petites cartouches destinées à la fabrication domestique de crème chantilly. Sous le nom de "proto", il est consommé au cours de manifestations festives en extérieurs, ou privées à domicile. Les sources de gaz sont les bouteilles à usage industriel ou médical, et les cartouches. Il est fréquemment associé à des stupéfiants et à de l'alcool.

Un travail de prévention est donc nécessaire, pour éviter que la fréquence de cette utilisation détournée n'augmente.

\section{MOTS-CLÉS}

Protoxyde d'azote, toxicomanie, toxicité, analyse.

\section{SUMMARY}

Medicine makes widely use for nitrous oxide for his analgesic and anaesthetic properties for a long time. It is used as a propellant aerosol in food industry. Today in France, there is more and more misuse of this product, like in USA or in UK since twenty years. To alert the health professionals, the Lille Poison Center realized a synthesis of acute and chronic toxicity's data, and of nitrous oxide's detection and determination in biological samples. The scientific synthesis was realized from a bibliographical research with MEDLINE. Moreover, we were in touch with a local raver's association which gave more information about its consumption, particularly about amount, frequency or association with other toxic substances. Nitrous oxide is conditioned pure in liquid form or in a mixture with the same amount of oxygen in gaseous form. Those forms are conditioned in tanks of different sizes, depending on the firm. The color is function of the medical or industrial use. Little cartridges for household whipped cream fabrication are available. Nitrous oxide is consumed during private or rave-parties. Origin of the gas are stolen tanks (industrial or medical use) or cartridges. It is frequently consumed with other drugs and alcohol. prevention is needed to avoid an increase of this misuse.

\section{KEY-WORDS}

Nitrous oxide, addiction, toxicity, analysis. 


\section{Introduction}

De nombreux produits, normalement réservés à l'usage médical, sont utilisés hors de leur contexte thérapeutique et de façon illicite, souvent associés à d'autres stupéfiants. Ainsi, le protoxyde d'azote fait l'objet d'un usage détourné qui semble se développer dans les milieux habituellement consommateurs de stupéfiants classiques. En effet, le Centre Antipoison de Lille a reçu depuis septembre 1999 plusieurs appels au sujet du protoxyde d'azote consommé au cours de rave-parties.

Afin de sensibiliser le corps médical, de faciliter le diagnostic et la prise en charge thérapeutique des intoxications à ce produit, et de mener des actions de prévention et de réduction des risques, nous avons réalisé une synthèse des données sur la toxicité aiguë et chronique du protoxyde d'azote. Nous présentons également les données récentes sur sa détection et son dosage dans les milieux biologiques.

\section{Méthodes}

La synthèse des données scientifiques a été réalisée a partir d'une recherche bibliographique utilisant MedLine (mots clés : nitrous oxide - adverse effects abuse - poisoning - death - analytical determination), et à partir des données fournies par les laboratoires pharmaceutiques et industriels qui distribuent le gaz, notamment les résumés des caractéristiques du produit (RCP).

Par ailleurs, des contacts avec une association lilloise de ravers ont permis d'avoir des connaissances plus pratiques quant à la manière de consommer ce produit en terme de lieu, quantité, fréquence et associations avec d'autres produits.

\section{Historique, présentation, usage du Protoxyde d'Azote} 1772 : synthèse par Priestley,

1800 : découverte de ses effets analgésiques par Davy, 1844 : application à la dentisterie, par Colton et Wells, 1868 : premier mélange du protoxyde d'azote à l'oxygène, par Andrews,

1881 : première utilisation pour soulager la douleur du travail chez une parturiente, par Klikovitch,

Fin du XIX siècle : large utilisation du gaz pur en anesthésie et en dentisterie,

1960 : n'est plus réservé au bloc opératoire,
1961 : première préparation d'un mélange fixe 50/50 de protoxyde d'azote et d'oxygène dans un même cylindre. Nom commercial : ENTONOX ${ }^{\circledR}$.

Depuis, large utilisation de ce mélange dans les ambulances et les services d'urgences, surtout au RoyaumeUni (1).

Le protoxyde d'azote est un gaz incolore, inodore, sans saveur ou légèrement sucré. Il a pour formule $\mathrm{N}_{2} \mathrm{O}$, et pour synonymes hémioxyde d'azote, oxyde nitreux, nitrous oxide. Dans le langage de la rue, c'est le proto. Il a deux domaines d'application : L'un médical (anesthésie, analgésie), sous forme d'un mélange avec de l'oxygène, l'autre industriel quand il est pur.

Il est utilisé depuis la fin du XIX ${ }^{\text {eme }}$ siècle en complément d'une anesthésie, pour son induction et son maintien (2). Dans ce cas, il est employé à forte concentration : toujours supérieure à $66 \%$, (concentration à partir de laquelle est observée la perte de conscience (3)), mais toujours inférieure à $80 \%$ (concentration au-delà de laquelle les dommages causés par l'hypoxie sont irréversibles (4)).

Il est utilisé en analgésie sous forme d'un mélange équimoléculaire d'oxygène et de protoxyde d'azote (MEOPA). Au Royaume-Uni, pionnier de la méthode, il est utilisé massivement dans les ambulances, dans les services d'urgence et les salles de travail depuis le début des années soixante, et en France depuis vingt ans dans le transport médicalisé et dans les salles d'accouchement.

Dans l'industrie alimentaire, il est utilisé comme gaz propulseur (crème chantilly). Il est aussi vendu sous forme de petites cartouches pour siphon (chantilly, eau de Seltz). Il est utilisé dans l'industrie mécanique de précision pour le nettoyage des petites pièces (horlogerie, photographie). Dans les sports mécaniques, aux Etats Unis d'Amérique notamment, il est utilisé pour refroidir les moteurs tournant à très haut régime, comme ceux des dragsters.

Le gaz simple est conditionné sous forme liquide, alors que le mélange $\mathrm{N}_{2} \mathrm{O} / \mathrm{O}_{2}$ reste gazeux, ce qui implique certaines contraintes de stockage. Ainsi, soumis à des températures inférieures à $-5^{\circ} \mathrm{C}$ pendant plus de 15 minutes, le protoxyde d'azote au sein du mélange se liquéfie et tombe au fond de la bouteille, exposant le patient à une inhalation d'un mélange non pas équimoléculaire mais d'abord très riche en oxygène puis de plus en plus concentré en protoxyde d'azote. Un risque important d'inhalation de protoxyde d'azote pur avec de graves conséquences hypoxiques est donc encouru par le patient. Les bouteilles de MEOPA doivent donc 
impérativement être stockées entre $0^{\circ} \mathrm{C}$ et $50^{\circ} \mathrm{C}$. Les laboratoires fabricants recommandent également de rouler la bouteille avant utilisation pour pallier la démixtion du mélange (5).

Le gaz est conditionné dans des bouteilles de taille et capacité variable, de type B5 à B20. Une fois remplies, les bouteilles ont des masses respectives voisines de $12 \mathrm{~kg}$ et $60 \mathrm{~kg}$. Le stockage s'effectue à la verticale dans un local fermant à clef.

Le protoxyde d'azote médical pur est un médicament réservé à l'usage hospitalier. Il est inscrit sur la liste II des substances vénéneuses et sa demande d'Autorisation de Mise sur le Marché est en cours.

Le MEOPA est également inscrit sur la liste II des substances vénéneuses et il est depuis le 30.03 .98 sous le régime d'une Autorisation Temporaire d'Utilisation de type cohorte. Celle-ci n'a pas pour objectif de mettre en évidence les propriétés thérapeutiques, parfaitement reconnues, du mélange, mais plutôt d'en définir les conditions d'un meilleur usage.

\section{Indications médicales, mode d'action, effets secon- daires et contre-indications du Protoxyde d'Azote}

Il est utilisé en association avec d'autres anesthésiques pour l'induction et le maintien de l'anesthésie, toujours mélangé à l'oxygène. En analgésie on l'utilise pour tout geste nécessitant une analgésie de moins de trente minutes : soins au cours de l'aide médicale d'urgence, ponctions lombaires et de moelle osseuse, réductions de certaines fractures simples ou de luxations périphériques, soins des plaies superficielles et des brûlures peu étendues, ponction veineuse chez l'enfant. Il est indiqué chez l'adulte et l'enfant de plus de quatre ans (5). Chez l'enfant plus jeune, l'administration devra être effectuée par un médecin anesthésiste ou familier de la méthode (6).

Le protoxyde d'azote n'est pas métabolisé par l'organisme. Il est absorbé et éliminé très rapidement par voie pulmonaire: Aucun chiffre de demi-vie d'absorption et d'élimination n'est pour l'instant disponible. Il est retrouvé sous forme inchangée dans l'air expiré, le sang et les urines.

Son mécanisme d'action n'est pas encore clairement connu, mais de nombreux auteurs évoquent une interaction avec le système opioïde endogène, le protoxyde d'azote pouvant être un agoniste partiel des récepteurs opiö̈des mu, kappa, sigma et delta (7). De plus, l'administration d'antagonistes spécifiques comme la naloxone atténue les effets du protoxyde d'azote (8).

Les avis sont partagés sur son éventuel potentiel addictif. Pour Gilman il est évident mais très faible, comparé aux autres substances addictives (9). Cet auteur insiste sur la fréquence des associations à d'autres substances addictives, ce qui ne permet pas de conclure formellement à son potentiel toxicomanogène, même après deux siècles d'utilisation (10).

Le protoxyde d'azote est un dépresseur du système nerveux central. De plus, il va prendre progressivement la place de l'oxygène avec pour conséquence, en l'absence de surveillance médicale ou à trop fortes concentrations, l'asphyxie voire le décès par arrêt respiratoire. Toutefois, dans le respect de ses indications et en l'absence de contre-indication, les effets secondaires sont rares et réversibles immédiatement à l'arrêt de l'inhalation : euphorie, rêve, paresthésies, agitation, nausées, vomissements. Les deux premiers effets sont ceux qui ont motivé son utilisation détournée.

Les contre-indications sont connues et doivent être respectées pour un bon usage : traumatisme crânien non évalué, toute altération de l'état de conscience, hypotension intracrânienne, traumatismes maxillofaciaux, pneumothorax non draîné, distension gastrique ou intestinale, température ambiante inférieure à $-5^{\circ} \mathrm{C}$ pour le MEOPA (5).

Dans le respect de ces indications, le protoxyde d'azote et le MEOPA en milieu médicalisé se sont révélés des médicaments très efficaces et d'une grande sécurité depuis respectivement deux siècles et trente ans.

\section{Détection et dosage dans les milieux biologiques}

La littérature scientifique est assez pauvre à ce sujet, mais c'est plutôt du à la rareté des intoxications aiguës depuis plus de vingt ans. Les dosages se font toujours par chromatographie en phase gazeuse (CPG).

En 1977, DiMaio et Garriott proposaient de dégazer du sang $(10 \mathrm{ml})$ par de l'air et d'analyser l'espace de tête du flacon par CPG avec un détecteur thermoionique (11). Dans deux cas de décès consécutifs à un usage abusif, les concentrations retrouvées étaient de $126 \mu \mathrm{g} / \mathrm{ml}$ et $143 \mu \mathrm{g} / \mathrm{ml}$. En 1992, Wagner et col. analysaient l'air prélevé directement à la seringue après clampage des poumons et des bronches (12). L'analyse de l'échantillon fut réalisée par CPG couplée à une 
détection par spectrométrie de masse Il n'était fait état que de l'identification du protoxyde d'azote dans l'espace de tête du flacon, et aucune concentration n'était donnée. Récemment, Poli et col. ont utilisé une microextraction en phase solide à partir d'un échantillon d'urine. Le dosage est effectué en CPG sur une colonne capillaire constituée d'un polymère poreux de divinylbenzène, avec détection par spectrométrie de masse. La limite de détection était de $100 \mathrm{pg} / \mathrm{ml}$ : elle permet ainsi la surveillance de l'exposition chronique de travailleurs exposés au protoxyde d'azote (13).

\section{Utilisation détournée}

L'utilisation détournée du protoxyde d'azote est décrite aux USA depuis plus d'une vingtaine d'années aussi bien chez des professionnels de santé (14) que chez des usagers extérieurs au milieu médical (15), alors qu'elle n'a été mise en évidence en France que très récemment. C'est à ce niveau que des contacts avec une association lilloise de ravers nous ont permis d'obtenir des renseignements pratiques sur l'utilisation détournée de ce produit.

Le protoxyde d'azote est rebaptisé "proto" dans le langage de la rue. Il est consommé au cours de manifestations festives comme les free-parties ou les technivals ainsi que dans des soirées privées. Les free-parties sont des soirées organisées en extérieur rassemblant deux cents à trois cents personnes en moyenne, annoncées par des flyers que l'on peut trouver chez des disquaires ou dans des cafés spécialisés. Ces flyers sont des supports publicitaires de la taille d'une carte postale, sur lesquels figure un numéro de téléphone correspondant à une boîte vocale indiquant les lieux et horaires du rendez-vous, le plus souvent, un parking de centre commercial. De là, les participants sont guidés par une voiture qui connaît le lieu choisi pour la fête, jusqu'alors tenu secret, et qui va les y conduire. Sur place se trouvent, outre la musique techno, alcool, nourriture et psychotropes divers : cannabis, LSD, psilocybes, cocaïne, kétamine, acide gammahydroxybutyrique, des amphétamines dont l'ecstasy et enfin, le "proto".

Les technivals sont des manifestations festives oganisées sur le même principe que les free-parties mais pour une durée de plusieurs jours (un à six) et rassemblant jusqu'à plusieurs milliers de personnes. C'est lors de l'une de ces technivals en septembre 1999 que le proto a été signalé.

Les bouteilles sont volées par effraction dans les laboratoires, pharmacies centrales ou cliniques qui stockent le produit. Sur le lieu de la fête, la bouteille est ensuite vidée dans des ballons de baudruche ou des préservatifs. Il arrive malheureusement qu'en l'absence totale de contenant, l'inhalation se fasse directement à la bouteille, exposant l'individu à de graves risques de gelures de la cavité buccale ou des voies aériennes supérieures (16).

Le "proto" est également consommé au cours de soirées privées dans lesquelles on utilise une autre présentation du gaz : ce sont des petites cartouches vendues en grande surface comme propulseur de chantilly pour un prix moyen unitaire de cinq francs, qui fournissent le protoxyde d'azote. Chaque cartouche mesure environ $5 \mathrm{~cm} \times 1,5 \mathrm{~cm}$ et peut fournir environ cinq litres de gaz pur. L'utilisation la plus fréquente est le remplissage de ballons ou de préservatifs, mais l'inhalation peut se faire directement à partir de la cartouche.

Que ce soit lors de manifestations festives en extérieurs ou privées, le faible coût de ce produit, associé à une cinétique très rapide d'apparition et de disparition des effets recherchés, a pour conséquence de pousser à une consommation excessive. Certains individus pourront ainsi inhaler une trentaine, voire une cinquantaine de ballons par soirée.

\section{Intoxication aiguë}

La gravité du tableau clinique de l'intoxication aiguë consécutive à un usage détourné est fonction de la dose absorbée et des substances associées. Au-delà d'une concentration de $66 \%$, on observe une perte de conscience, exposant l'individu à des risques de chutes graves. De même, à cette concentration en protoxyde d'azote, les réflexes de toux et de protection du Iarynx ne sont plus conservés (3). La durée de l'inhalation est en moyenne de trente secondes pour un ballon. La répétition des prises est favorisée par un faible coût du gaz. Les substances associées sont nombreuses dans ce type de manifestations. Un risque majeur est encouru avec celles qui, comme le protoxyde d'azote exercent une action dépressive puissante sur le système nerveux central (alcool, benzodiazépines).

Les complications majeures de l'intoxication aiguë sont bien identifiées. Le décès peut être rapide par hypoxie. En 1971, une revue de la littérature comptabilise un décès unique au cours des vingt dernières années (17). Puis, les cas se multiplient chez les professionnels de santé $(18,19)$. En 1990, Suruda et Glothlin signalent onze décès survenus de 1984 à 1987 suite à des usages abusifs par des professionnels de l'industrie alimentaire (20). Après 1985, des cas de décès dans les manifestations festives sont décrits $(12,21)$. 
La deuxième complication est représentée par l'inhalation de vomissements. En effet, les vomissements, initialement effet indésirable propre au protoxyde d'azote inhalé à dose élevée, sont favorisés par l'association de substances émétisantes comme l'alcool. Le danger est majoré si la concentration en protoxyde d'azote est supérieure à $66 \%$, privant l'individu de sa conscience et de ses réflexes de toux et de protection laryngée et l'exposant ainsi plus fortement à un risque d'inhalation.

\section{Intoxication chronique}

Il n'existe pas, pour l'instant, de dose toxique à long terme qui soit clairement définie. On observe des myéloneuropathies tardives lors d'expositions chroniques à fortes doses (22) lors d'utilisations médicales (23), comme lors d'utilisations détournées (24). Les symptômes initiaux peuvent apparaître de façon différée et prendre la forme de faiblesse des membres inférieurs, de troubles de l'équilibre, de paresthésies des extrêmités et du tronc, éventuellement d'une positivité du signe de Lhermitte (sensation de décharge électrique lors d'une flexion antérieure de la nuque). Ces troubles sont susceptibles de se manifester dans les six mois qui suivent l'arrêt de l'utilisation chronique (25). Certains auteurs incitent d'ailleurs à un bilan neurologique systématique en cas de suspicion d'usage abusif de protoxyde d'azote (26). Des cas d'anémie mégaloblastique sont également signalés lors d'expositions prolongées ou répétées (27). Le mécanisme d'action de ces deux effets serait une oxydation de la vitamine B12 par le protoxyde d'azote. Celle-ci, ainsi rendue inactive, peutêtre supplémentée par des administrations d'hydroxocobalamine. Ce type de traitement est d'ailleurs à la base de la résolution définitive des troubles neurologiques induits par une consommation excessive du gaz (26).

\section{Conclusion}

Le protoxyde d'azote à usage médical bénéficie d'une grande efficacité et d'une haute sécurité en anesthésie et en analgésie, chez l'adulte comme chez l'enfant et ce depuis de nombreuses années. Cependant, dès que les conditions d'utilisation ne sont plus respectées, il peut conduire à des intoxications aiguës avec risque de décès et des effets néfastes à long terme. Nous voulons donc alerter les professionnels de santé sur les dangers de l'utilisation détournée et abusive du protoxyde d'azote en France, comme c'est déjà le cas depuis longtemps au Royaume-Uni et aux Etats-Unis.
Un travail de prévention reste donc à effectuer, sur le terrain comme auprès des professionnels de santé, afin que ce médicament n'échappe plus à son contexte. Cela pourrait passer par une meilleure surveillance ou une réglementation plus sévère des conditions de stockage et de distribution, principales sources d'approvisionnement du circuit parallèle.

\section{Remerciements}

Sincères remerciements au Docteur Jean-Christophe LINKE pour son aide précieuse et sa patience admirable. 


\section{Références}

1. Carbajal R., Analgésie par mélange (50/50) de protoxyde d'azote/oxygène chez l'enfant. Arch. Pédiatrie 1999; $6: 578-85$.

2. Liénart A, Protoxyde d'azote. Encycl. Méd. Chir., Anesthésie-Réanimation. $1990 ; 36279$ A $10: 13 p$.

3. Parbrook G.D., Therapeutic uses of nitrous oxide. Br. J. Anaesth. $1968 ; 40: 365-72$.

4. Rogo E.J., Lupovici E.M., Nitrous oxide - an occupational hazard for dental professionals. Dent. Hygiene $1986 ; 60$ : 508-514.

5. Résumé des caractéristiques du produit (mélange équimoléculaire d'oxygène et de protoxyde d'azote). Laboratoire Air Liquide Santé.

6. Annequin D., Ethique ou réglementation. La difficulté du choix. Le cas du mélange protoxyde d'azote oxygène. La lettre de Pédiatrie. $1998 ; 5: 1-12$.

7. Gilman M.A., Nitrous oxide, an opioïd addictive agent. Revew of the evidence. Am. J. Med. 1986 ; 81 : 97-102.

8. Gilman M.A., Analgesic (subanesthesic) nitrous oxide : interacts with the endogenous opioild system. Life Sci. $1986 ; 39: 1209-21$.

9. Gilman M.A., Analgesic nitrous oxide in neuropsychiatry : past, present, futur. Int. J. Neurosci. $1989 ; 49: 75-81$.

10. Gilman M.A., Nitrous oxide : abuse in perspective. Clin. Neuropharmacol. $1992 ; 15: 297-306$.

11. DiMaio V.J.M., Garriott J.C., Four deaths resulting from abuse of nitrous oxide. J. Forensic Sci. 1977 ; 23 ; 169-72.

12. Wagner S.A., Clark M.A,Wesche D.L., Doedens D.J., Llyod A.W., Asphyxial death from the recreational use of nitrous oxide. J. Forensic Sci. 1992 ; 37 : 1008-1015.

13. Poli D., Bergamaschi E., Manini P., Andreoli R., Mutti A., Solid-phase microextraction gas chromatographicmass spectrometric method for the determination of inhalation anesthetics in urine. J. Chromatrogr. B. 1999 ; $732: 115-25$.
14. Rosenberg H., Orkin F.K., Springstead J., Abuse of nitrous oxide. Anesth. Analg. $1979 ; 58$ : 104-6.

15. Sterman A.B., Coyle P.K., Subacute toxic delirium following nitrous oxide abuse. Arch. Neurol. $1983 ; 40: 446-7$.

16. Hwang J.C., Himel H.N., Edlich R.F., Frosbite of the face after recrational misuse of nitrous oxide. Burns. $1996 ; 22: 152-3$.

17. Smith W.D.A., Pharmacology of nitrous oxide. Int. Anesth. Clin. 1971;9:91-123.

18. Chadly A., Marc B., Barres D., Durigon M., Suicide by nitrous oxide poisoning. Am. J. Forensic. Med. Pathol. $1989 ; 10: 330-1$.

19. Winek C.L., Wahba W.W., Rozin L., Accidental death by nitrous oxide inhalation. Forensic Sci. Int. $1995 ; 73$ : 139-41.

20. Suruda A.J., Mc Glothlin J.D., Fatal abuse of nitrous oxide in the workplace. J. Occup. Med. $1990 ; 32: 682-4$.

21. Gowitt G.T., Hanzlick R.L., Atypical autoerotic deaths. Am. J. Forensic Med. Pathol. 1992 ; 13 : 115-9.

22. Layzer R.B., Myeloneuropathy after prolonged exposure to nitrous oxide. Lancet. $1992 ; 2: 1227-30$.

23. Pema P.J., Horak H.A., Wyatt R.H., Myelopathy caused by nitrous oxide toxicity. Am. J. Neuroradiol. $1998 ; 19$ : 894-6.

24. Lunsford J.M., Wynn M.H., Kwan W.H., Nitrous oxide induced myeloneuropathy. J. Foot Surg. $1983 ; 22: 222-5$.

25. Heyer E.J., Simpson D.M., Bodis-Wollner I., Nitrous oxide : clinical and electrophysiologic investigation of neurologic complications. Neuroly $1996 ; 36: 1618-22$.

26. Brett A., Myeloneuropathy from whipped cream bulbs presenting as conversion disorder Aust. N. Z. J. Psychiatry. $1997 ; 31: 131-2$.

27. Berger J.J., Model J.H., Sypert G.W., Megaloblastic anemia and brief exposure to nitrous oxide - a causal relationship. Anesth. Analg. 1988; 67 : 197-8. 